\title{
Students' learning outcomes from cross-collaborative supervision in information seeking processes during work placements
}

\author{
Peer reviewed \\ Anett Kolstad \\ University College of Oslo and Akershus
}

\begin{abstract}
This article presents findings from a study of student experiences of collaborative teaching and learning outcomes related to information literacy (IL) and evidence-based practice (EBP) following interdisciplinary supervision of their assignments by nurse educators, nurse supervisors and librarians in real clinical settings.

The article is based on qualitative and quantitative text analysis of 102 individual student logs, qualitative text analysis of 36 student group assignments, feedback from an evaluation form and 285 blog and wiki comments from students, nurse educators, nurse supervisors and librarians. It is analysed according to the first five steps of the EBP model and feedback from an evaluation form.

The students' learning outcomes in information literacy improved by using the EBP model. By the end of the project period, $83 \%$ of the students had integrated a focus on research-based knowledge into their work placement assignment. The interdisciplinary joint supervision and a related blog and wiki communication forum had significant influence on this outcome area. The preparation programme for the students on campus, before work practice placements was developed collaboratively between the nursing education programme and the Learning Centre and library.

There is very little existing research on the effect of cross-collaborative supervision in IL where both physical and digital tools have been used in work placements. This challenges established routines and ways of conducting supervision in IL in both the library and nursing education, because of the need to collaborate more tightly than before.
\end{abstract}

Keywords: Information literacy, information seeking, evidence-based practice, cross-collaborative supervision, nursing work placements

\section{Contact:}

Anett Kolstad

University College of Oslo and Akershus, Norway

e-mail:kolstadakk@gmail.com 


\section{Introduction}

The Learning Centre at Oslo University College in Norway had taught students information literacy (IL) as a standalone subject up until autumn 2009. Then, the nursing degree programme invited the Learning Centre to participate in supervising about 400 undergraduate nursing students (first year Bachelor's degree), 50 at time, in one of the largest work placement initiatives in Oslo, the Langerud project. The nursing degree programme wanted to engage a librarian to teach students IL to acquire research-based evidence as part of evidence-based practice (EBP) in the project that was finished in the spring of 2012. According to the Langerud project, the 50 students were to be trained by nurse educators, nurse supervisors and librarians, through learning management systems (LMSs), such as blogs/wiki, and face-to-face at physical meetings in the work environment.

A sociocultural view implies that IL as a phenomenon is shaped by social interaction and variation between situations and contexts (Lloyd, 2013). The students and supervisors were to learn from each other in both physical and digital "meeting places", and the Learning management systems had a significant mediational role (Kolstad, 2012; 2015).

The aim of this article is to present findings from this project, based on the student experiences of collaborative teaching and learning outcomes in IL and EBP. It is primarily information seeking that is investigated in the project, but also how students would use the required information in work placements.

Research questions include:

- How did the students develop a set of abilities and understanding of information literacy in an EBP setting?

- How did the experiences of collaborative teaching and the students learning outcomes contribute to changes in preparing the students for practice periods in work placements?

The article initially describes the supervision culture in the Langerud project, presenting a literature review and the methods used to analyse the findings, based on the seven steps in the EBP model (Melnyk, Fineout-Overholt, Stillwell, \& Williamson, 2010) in combination with the influence of joint supervision. The findings are then discussed according to how the joint supervision culture in Leeway for information seeking (Kolstad, 2015), EBP requirements, and use of mediating tools (such as face-to-face supervision and/or online-supervision through LMSs) affected the students' results.

\section{Joint supervision culture in the Langerud project}

A joint supervision culture among nurse educators, nurse supervisors and librarians was developed and this was studied as part of a sub-project of the Langerud project, Leeway for information seeking (Kolstad, 2015). In this study the supervisors constituted an interdisciplinary supervisory team. The findings in the article were presented according to Wenger's understanding of communities of practice; shared repertoire, joint enterprise and mutual engagement (Wenger, 1998, p. 95). The joint supervision culture was characterised by personal and close communication, a culture of sharing, common supervision vocabulary, a focus on support to the students and acknowledgement of each other's competences. The issues discussed in the author's previous article (Kolstad, 2015) included relevance of content, subject terminology, information seeking processes, literature selection and how to integrate research findings into work placements. The librarian became part of the community of practice and 
adopted the common structures guiding how the collaboration and supervision should proceed. The librarian's teaching or supervision was totally integrated and became a catalyst for information literacy (IL) workplace learning. According to my previous article (Kolstad, 2015) IL lays the foundation for EBP. EBP requires that decisions about health care are based on the best available, current, valid and relevant evidence (Dawes et al., 2005; Sackett, Rosenberg, Gray, Haynes, \& Richardson, 1996).

In the Langerud project, it was crucial for students to learn how to "think evidencebased" and to manage five of the seven steps in the EBP (Melnyk et al., 2010). This implies an emphasis on cultivating a spirit of inquiry where students ask clinical questions in the PICOformat (Problem Intervention Comparison Outcome), search for the best evidence, critically appraise it and integrate it with clinical expertise and patient preferences and values. The supervisors had different roles depending on the type of problems that the student wanted to discuss or find an answer to. This will be further described in relation to the students' learning outcomes in the results section of this article. The way in which the supervision culture influenced the results will also be taken into consideration.

\section{A literature review of students' IL learning in work practice}

Research indicates that IL is most easily acquired in the clinical practice setting rather than stand-alone teaching (Coomarasamy \& Khan, 2004; Sundin, 2003). Both the systematic review of Coomarasamy and Khan and the study of Sundin confirm that skills, attitudes and behaviour are developed when IL is integrated in practice. Coomarasamy and Khan mention potential reasons for integrated teaching achieving better outcomes than stand-alone teaching. For example, the learner identifies a real clinical problem, evidence is sought actively, practical use of acquired knowledge is evident, case discussions are used to learn how to incorporate knowledge, information is directly relevant to practice and if the information is stored electronically it can be easily retrieved (p. 4). Several studies support that both having knowledge of EBP and taking part in EBP groups affect the development of a positive attitude to EBP (Stokke, Olsen, Espehaug, \& Nortvedt, 2014; Wallen et al., 2010). Lloyd (2013) argues that a librarian should have a mediating and supporting role for nurses in their first year in a workplace after qualifying. As mentioned previously, there is a recognised need to increase IL capacities and integrate evidence-based practices into workplaces. However, to achieve this, students also need to be better prepared for practice periods in work placements (Lloyd, 2013).

The participants in the Langerud project assumed that students could acquire knowledge about information seeking and the importance of medical databases during the nursing degree programme on campus, but that they are less able to acquire skills, attitudes and behaviour that integrate EBP, if they do not experience the applicability in practice.

Several studies have investigated face-to-face supervision, or only online-supervision and hybrid learning management systems (Cobus, 2009; Figa, Bone, \& Macpherson, 2009; Florea, 2008; Kraemer, Lombardo, \& Lepkowski, 2007). Florea (2008) presents an overview of studies that have investigated different tools from the supervisor's point of view. Studies by Kraemer et al. (2007), Cobus (2009) and Figa et al. (2009) focused on the learning outcomes of students. Kraemer et al. concluded that hybrid possibilities including both face-to-face and virtual supervision of students were superior to selecting either one or the other. Cobus compared two groups of students; one sharing their work in a LMS, while the other group did not. He found a higher engagement for nursing as a profession in the first group and that the students naturally acquired a greater degree of reflection about the work through the shared pooling of experiences. Figa et al. (2009) found that an online-course should be able to meet the needs of both face-to-face supervision and digital teaching through the same resource tool. 
As mentioned previously, there are several studies that cover how to prepare students for the nursing profession and the tools that are best suited for use on campus and in stand-alone courses, but there are few studies about the potential for preparing undergraduate students during work placements, through face-to-face and LMS interdisciplinary supervision.

\section{Research design}

The librarian, who is also the author of this article, was the project manager of Leeway for information seeking. She had a dual role being both researcher and practitioner simultaneously. As mentioned in Kolstad (2015, pp. 62-65) the subjective influence may have affected the results. The librarian as a researcher gained insights into the needs of the students and acquired useful experiences and understanding through communication with the other supervisors, but also by communicating and supervising the students. In the project, she followed the students closely by supervising both in meetings with the learning group at the workplace and in LMS over a period of eight weeks. On campus, the librarian did not follow up with the students to the same degree. However, in the work placement context, she may have also become so involved that it could be difficult to be critical. This may have been balanced out somewhat with the two-year time lapse between the end of the project (2012) and the systematic analysis of data (2014), allowing greater neutrality.

The empirical material was collected both formally and informally through participation in the work placement learning programme from 2010 to 2012. This article is based on a qualitative and a quantitative text analysis of 102 individual student logs in which the students described what they had learnt during the practice period, qualitative text analysis of 36 out of 38 student group assignments and 285 blog and wiki comments from students, nurse educators, nurse supervisors and librarians. The logs were a part of the students individual work in the work placement. The assignments analysed were not always complete texts, since they could be oral presentations, written papers or just a table of content to use for a presentation. Fiftynine of the blog/wiki comments were from students and these constitute the major part of the empirical material that this article is based on. A selection of quotes about information seeking and the assignment have been extracted. These illustrate different aspects of the results. The material is taken from six of eight work placements during the period from spring 2010 when the project started to use the LMSs until spring 2012. In addition, the analysis is based on an evaluation form feedback from spring 2010 (Kolstad, 2010).

Source quotations are coded as SG1-8 for student groups in each of the six practice periods, PM for project manager, NE1-4/5 for nurse educators, NS1-15/50 for nurse supervisors and L1-3/3 for librarians and the practice periods are coded as S2010(2), S2011 sometimes divided into S2011(1), S2011(2), S2012(1), A2010, A2011 for practice periods in spring (S) / autumn (A) and year. The quotations are translated from Norwegian into British English and are as directly translated as possible. The quotations from assignments and student logs are not tied to any specific student, to respect confidentiality and privacy.

For the assignment, the students were required to find and use research articles (and other literature) applying five steps of the seven step model of EBP as a structure for the work (Melnyk et al., 2010). In their logs, the students were asked to reflect on what they had learnt during the work placement, especially focusing on their IL learning, use of research articles and understanding of EBP. In the LMS, the students could ask questions, answer and discuss issues with their supervisors.

The material that consists of unstructured text has been sorted according to steps zero 
to four in the seven-step EBP-model.

The following explains what is expected of the students for each step:

\section{Cultivate a spirit of inquiry}

The students were encouraged to be inquisitive about how practices work and to ask critical questions in the following manner.

2. Asking clinical questions in the PICOT format

Inquiries in this format take into account patient population $(\mathrm{P})$, intervention $(\mathrm{I})$, comparison $(\mathrm{C})$, outcome $(\mathrm{O})$ and time $(\mathrm{T})$. The format provides an efficient framework for information seeking in medical science databases. For this study, the time-factor (T) was not required.

3. Searching for the best evidence

The search is streamlined when questions are asked in the PICO format. This helps to identify key words or phrases that, when entered successively and then combined expedite the location of relevant articles in databases, such as MEDLINE or CINAHL.

Sundin distinguishes between 'clinically oriented' information seeking and 'academic oriented' information seeking (Sundin, 2003, p. 173-5). A crossing between these two is the idea behind the approach to EBP in the assignment that the students were required to carry out. Clinically oriented, in the sense that it should be relevant to the workplace and the students should be able to defend it in front of their department in the workplace.

4. Critically appraising the evidence

The students were asked to consider whether the findings were valid and if they were relevant for the patient.

To evaluate this, the literature list was critically reviewed at this stage. The students were required to use different kinds of literature, but at least one up-to-date research article and the American Psychological Association style for references (2010).

5. Integrating the evidence with clinical expertise and patient preferences and values At the end of their work placement, the students were required to present an assignment to the department nurses, the nurse educator, the nurse supervisor and the librarian.

In the assignment, the students were asked to use the EBP-model and show that they had managed to combine the three aspects based on what they had learnt in the information seeking process and through their own experience (figure 1). This required information seeking in scientific databases to find research articles.

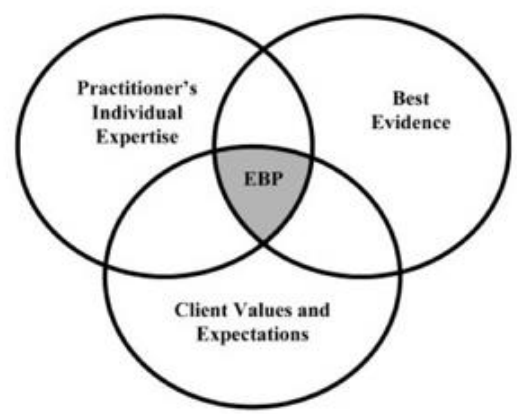

Figure 1. The EBP model 
This model was used to give the students a tool to 'think' evidence-based; to make them aware of the three aspects of EBP (Dawes et al., 2005; Sackett et al., 1996). The $102 \operatorname{logs}$ have been read and analysed according to all the three circles, to find out whether the students combined them and, if so, how many. Information has been extracted from the logs to establish how many students described the importance of being up-to-date with the best evidence and how they had searched for research articles. This was interesting, since the librarian's contribution was aimed at strengthening the focus on research-based practice in the EBP-model.

All 102 student logs have been analysed in order to explore students' approaches to the various aspects of the EBP model (figure 1) and how this was expressed. All related statements on this aspect were registered in an excel-form.

The last two steps have not been considered, because they were not naturally tasks for the students:

6. Evaluating the outcomes of the practice decisions or changes, based on evidence

7. Disseminating EBP findings

Additionally, the evaluation feedback form and the student logs have been used to find out how the students described the inputs of the supervisors and the importance of LMS as a mediating tool.

\section{Results}

\section{The set of abilities and understanding of information literacy in an EBP setting}

The students were used to presenting assignments. However, this project was different in that they had to describe the process; the selection of the focus issue, how they conducted the information seeking, their choice of subject terms, the sorting of the information into the PICO format throughout the assignment and specifically for the presentation. This step-by-step manner of reporting gave the students the opportunity to show how they were thinking and to ask questions from the interdisciplinary supervisory team on a more regular basis than usual (Kolstad, 2015). According to these steps, the main findings were as follows:

\section{Cultivate a spirit of inquiry}

All the students found relevant topics for their work placement. Reasons for selection of an issue included: the issue being of interest to the department, a topic the students found particularly interesting through their work experience or an issue where it was felt that further competence would be useful. An example of an issue was "What can we do to improve communication and socialising between patients?" (comment in LMS, SG1, S2010). Students thought that this issue was interesting, because they felt that patients were sitting silently around the same table without talking to each other. In some cases, the students identified a gap between what they had learnt in their nursing degree programme and the situation during the work placement. This motivated them to investigate an issue more closely. An example of an issue was "infection risks from jewellery" (assignment, S2010), because on campus the students had learned that, they shouldn't use jewellery at work.

The students were encouraged to reflect on their selection of an issue to explore them. They often discussed the issue with the nursing department they were assigned to during the practice period. In some cases, the department requested students to examine a specific issue in more detail, because they wanted to have more information about it (Kolstad, 2015, pp. 68-69). 
In other cases, the department helped the students find a relevant issue that they could work with. For example, they supported the students who had chosen the issue medication error by saying "I think this is an important issue, because it is easy to think that it is the responsibility of the doctor, but it is also the nurse's responsibility" (comment in LMS, NS9, SG4, A2011) or they gave support by saying "Your issue is very interesting" and taught the students more about their chosen issue (comments in LMS, NS2, NS5, S2010).

In several cases, the theme was chosen because the students themselves thought it was interesting or exiting. One learning group expressed this in the following manner, "We think this (the sensory garden project) is something new and interesting for us and something we believe in. The sensory garden should be used. We want to make it more attractive to patients, relatives and employees" (assignment, A2010). Another example was, "We have seen that nutrition is a relevant issue and the Norwegian Directorate of Health has just come out with new guidelines" (assignment, A2010). A third group had chosen sensory stimulation as an issue, because it was a major project during their work placement (comments in LMS, SG2, S2011).

The nursing supervisor sometimes encouraged the students in their choice of topic and demonstrated her professionalism in this way, "Hello! We think music is a great theme! Music can have different influences on different people. For this reason, it might make sense to consider using qualitative research" (comments in LMS, NS14, S2011).

\section{Asking clinical questions in the PICO format}

As regards students' choice of clinical questions, the librarian got particularly involved in matters regarding possibilities to find research-based information about an issue (Kolstad, 2015 , p. 69). She also interacted with students in relation to the choice of subject terms and structuring the problem into a PICO format. The nurse educator supported the librarian with regard to sorting terms in a structured way (Kolstad, 2015, p. 69).

The result was that most of the learning groups sorted their issues into the PICO format correctly and almost every learning group had found relevant subject terms (Medical Subject Terms) to use for the information seeking process. Nevertheless, the thoroughness of information seeking in the international databases varied significantly between groups.

In relation to finding subject terms, one of the students said, "It was more difficult than I thought to find correct terms, but in the work placement I have practiced a little and I'm working on becoming better. I have learned the importance of doing the information seeking correctly and finding trustworthy research to use" (student log, A2011).

One student described the PICO format in the following way: "An important tool for finding good research is using a model called Pico. One selects important aspects of what to search for in order to find the right research" (student log, S2011).

Here is an example of sorting subject terms correctly into a PICO-form:

\begin{tabular}{|l|l|l|l|}
\hline P & I & C & O \\
\hline Dementia & Stimuli of senses & & Positive experience \\
Alzheimer & Healing garden & & \\
\hline
\end{tabular}

Table 1: PICO-form (assignment, SG4, S2011(1)) 
Some students dropped the PICO format and formulated the issue according to the subject terms in Norwegian in the first row and in English in the second and third rows:

\begin{tabular}{|l|l|l|l|l|}
\hline Eldre & Demente & Sykepleie & Urinveisinfeksjon & Sykehjem \\
\hline $\begin{array}{l}\text { Elderly } \\
\text { Aged }\end{array}$ & Dementia & Nursing & $\begin{array}{l}\text { Urinary Tract } \\
\text { infection }\end{array}$ & $\begin{array}{l}\text { Nursing } \\
\text { homes }\end{array}$ \\
\hline
\end{tabular}

Table 2: Assignment table example without reference to the letters PICO (assignment, SG2, S2010)

The PICO format explains how students were thinking in terms of the purpose of focus on their issue and table 2 shows more how the students sorted the subject terms before starting the information seeking in international databases. The 'table-format' without thinking about the letters PICO, may have been easier to understand for some students, because it is about finding synonyms to subject terms and for searching in a certain way using 'or' between the synonyms in a column and 'and' between the results for each column, (see also the example in point 2 below).

3. Searching for the best evidence

It was not a requirement that students find the 'best' research, but rather that they start the exercise in the first practice period. There were some discrepancies between learning groups' thoroughness with the information seeking process in conjunction with the tasks. This may to some extent be a result of the disagreement between supervisors about the aim to carry out the information seeking in a certain way (Kolstad, 2015, p. 75).

It seemed that some students were not thorough in the information seeking process and did not use the PICO form as a starting point. They simply described, which words they had used and where they had been searching; "We did the information seeking in Medline and used the words 'MRSA', 'nursing homes' and 'elderly'. We did not remember the whole information seeking process, but this was mainly what we did" (comment in LMS, SG8, S2010). This group used an article from a Norwegian book in their assignment and may have found international articles too challenging.

Most of the learning groups were very thorough in their information seeking process. They used the PICO format as the starting point, chose several synonyms for one concept and combined it with synonyms for another concept. They also refined their information seeking, when they did not find relevant research articles.

An example from one of the learning groups (assignment, SG3, S2010): "First we did a simple search on urinary tract infections. To refine the quality of our findings, we did another search, like this:

1. $\exp$ Dementia/

2. exp Nursing/

3. exp Urinary Tract Infections/

4. exp Nursing Homes/

5. 1 and 2 and 3 and 4

6. 3 and 4

7. 2 and 6

8. from 7 keep $1-5$

9. limit 7 to (English language and humans and yr="2004 -Current" and ("all aged (65 and over)" or "aged (80 and over)"))

10. Find similar to Urinary Tract Infections among the institutionalised older adult". 
The same learning group presented their results from selecting resources: "We found one research article that we will use in our work: 'Urinary tract infection among the institutionalized older adult'. Perspectives: 28 (2): 23-7. 29-34, 2004. Blais, Dawn" and "Additionally, we have been conducting searches in Svemed+ with the search terms 'prevent' and 'urinary tract infection"”.

The information sources all students used to find relevant literature resources included the website Norwegian Health Library, various other Norwegian websites and Net encyclopaedias, the Google search engine, the databases Cinahl, Medline, Ovid Nursing, Svemed+, Pubmed, Bibsys and the institutional systems Gerica and Practical Procedures in Nursing services (comments in LMS, S2010, S2011, S2012). They also used literature from the syllabus. The librarian asked for some explanation for choices of databases (comments in LMS, L1, S2010, S2011 (1,2), S2012). However, in general, very little explanation was given. The assignments showed that the students were aware of the importance of the information seeking process to find research articles during the work placements. The logs contained descriptions of the students own reflections on their learning outcomes "We have been doing information seeking to find relevant research articles for the department presentation. In the beginning it was difficult, but now we have had a chance to practice a lot" (student log, A2010). Another example was "During my work placement I have spent a lot of time doing information seeking about different diagnoses and procedures. I have, for example, read the journal of my patient and done information seeking on her diagnosis to have a better insight into her situation. I have been searching mostly in the Norwegian Health Library, but also in databases, such as Svemed+ and Cinahl"' (student log, SG4, S2011).

4. Critically appraising the evidence

Most of the learning groups did not describe their critical appraisal of the evidence. May be they did not feel qualified to do this being undergraduate students in their first year of the Bachelor's degree in nursing. Nevertheless, some typical appraisals are mentioned below.

Although almost all learning groups described how they had sought and found English subject headings and applied them in searches in international databases, there were only a few, who had used up-to-date research articles in English. Most of the learning groups used research articles in Norwegian or other Scandinavian languages.

In one of the examples also referred to above, the students explained that they had done the information seeking in Medline and Svemed+. They had refined their search, because they did not find any relevant research articles. Among the articles this group found, was a dated article from 1992 (assignment, SG2, S2010). This indicates that they were not sufficiently critical of their research sources. When they presented the findings of the study in the article, it generated discussions in the department, because the findings were so dated (notes, S2010).

Some of the groups found it difficult to find up-to-date research on their issues. One example was the issues relating to the Sensory Garden mentioned above. To generate information they questioned and interviewed other professionals in addition to finding some research articles. Other groups tried to find something although it was difficult.

Some student groups evaluated their findings and gave arguments for rejecting some article as "... not so much for us to use, because it contained just a few irrelevant findings...". They also described why they included other articles.

Several groups asked questions about whether they could use sources from the syllabus and sought critical feedback from the supervisors (examples from all the practice periods, S2010S2012). This was also part of critical appraisal at this level, even if it was not necessarily 
research articles that they appraised.

5. Integrating the evidence with clinical expertise and patient preferences

One could see that the students had chosen issues relevant to their work placement, either because it was a specific request from the department, or the issue was of particular interest to them, as mentioned previously in point 1 . In the assignment presentations, the students were required to argue why they had used specific research references and to be able to connect them with clinical expertise, experiences and patient preferences.

Most of the learning groups highlighted the rationale for choosing a particular topic related to the individual departmental needs or patient needs and the interests of the learning group, as mentioned above. Based on these needs, they described how research findings could be useful for improving practise. The students used several presentation techniques, including straight oral presentations, a combination of oral and written presentations, PowerPoint-presentations, YouTube clips and self-made video films (comments in LMS, S2011-S2012).

In the example below, the learning group showed how they wanted to include those involved in the work placement when they presented the assignment to the department; "We have thought that I and NN will start with a presentation of the issue and research about over medication, its extent and consequences. After that the others are going to give a short presentation about the different focus areas. At the end, we would like to involve the nurses in discussing the issues by asking questions. We hope it will be educational for us and the employees!" (comment in LMS, SG3, A2011).

The supervisor's evaluation of student presentations included something about how they found that the students managed to connect all aspects of EBP, also mentioned in Kolstad (2015, p. 70), but with fewer examples. This feedback was usually shared on the LMS following the student presentations. Here is one example from a nurse supervisor "It was a very well planned and implemented input on how we as health professionals can prevent the spread of Staphylococcus aureus in wounds in a nursing home. Many useful views emerged during the discussion after the presentation and may lead to further development of the procedures we have" (comment in LMS, N15, SG5/6, A2010). The librarian gave positive feedback to a learning group, which had acquired information in several ways. They had conducted interviews with other workplaces and read various books and research articles. They had also used their own experiences (e.g. with the Sensory Garden) and listened to reflections about the patient's needs and wishes. They proposed several new tips for both patients and employees (assignment, A2010). There was a feedback comment from the nurse educator, stating that the content of a certain presentation was of a "high academic level". The presentation referred to explained how patient safety had been considered, the information seeking process and review of new relevant research. It was recommended as a project that everyone could benefit from reading (comments in LMS, NE1/PM, S2010).

Students were constantly encouraged to think evidence-based by reflective thinking (Kolstad, 2015, p. 76). One of tutors (L1/PM) tried to get students to reflect on the EBP-model. She claimed that the students would be able to respond to the content of each of the "circles" (figure 1).

One student description in a log showed how they understood EBP, "I have acquired research-based knowledge from relevant articles and research papers through databases and used the relevant literature. I have gained experiential knowledge by observing and performing various procedures, interacting with patients and learning by doing. I have understood user knowledge and user interaction by getting to know and listening to each patient. Professional ethics are very important and protect the patient's values. All these three aspects in knowledge- 
based practice are equally important and I have gained new knowledge in all aspects. Everything is connected and important to do in optimal nursing" (student log, A2010).

In the logs, the students described their learning outcomes. Almost two thirds of the students described all the three aspects of the EBP model. $39 \%$ described one or two aspects (fig. 2). Of these, just one had described research-based knowledge. Most of them had described both userbased knowledge and experience-based knowledge in the same log. $60 \%$ of the $102 \operatorname{logs}$ contained descriptions of research-based knowledge.

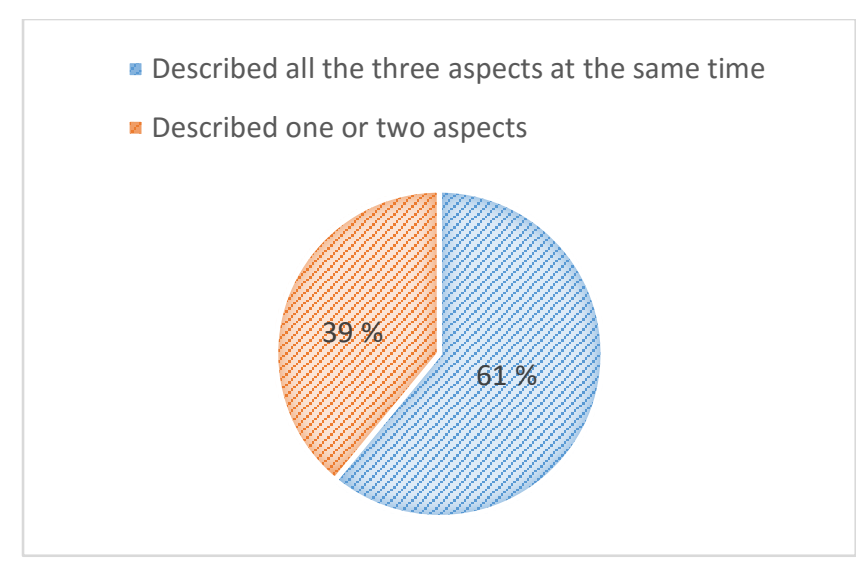

Figure 2. The number of students, who described all the three aspects of EBP or one or two aspects (Excel form, June 2, 2014)

One of the eight departments was distinguishable from the others, because the students delivered twice as many log entries as other departments. In total, the students from this department represented $25 \%$ of all the log entries. Each contained descriptions of all three aspects of the EBP model. This was the one significant difference between this department and the others, where it seemed that the students chose which aspect of EBP they wanted to focus on. The librarian participated in the learning group in the outstanding department in 2010, at the beginning of the project period, and one can see a correlation between this participation and the students' learning outcomes. Much emphasis was given to information seeking during the project period in this department. A statement from one of the students confirms, "There's no doubt that research has been an illuminated theme when we have been engaged in the work placement" (student log, S2011). Another similar comment confirms this "I also learned how the aspects of the EBP model are interrelated and influence each other, to provide the best possible health care needs all the five areas working together. I feel I have gained some knowledge from all of these areas and look forward to learning even more" (student log, S2011).

Before the project Leeway for information seeking started, the focus on research-based knowledge was very low or non-existent. The work to increase this focus started in the autumn of 2009. The student log entries describing their learning outcomes became a summary of the whole eight week practice period. The description of the information seeking process and focus on research-based knowledge varied. If the students mentioned if, how and why, they had used research articles they had a focus on research-based knowledge. Some of them also included comments about how the PICO format had been used, the information seeking process, choice of databases and choice of literature. The number of students, who described this in their log entries, was counted. In $\log s$ that were entered on the LMS in the spring of 2011, the focus on research-based knowledge had increased to $46 \%$. There was a further increase by the autumn of $2011(60 \%)$ and finally an increase to $83 \%$ by the end of the project period (see figure 3 ). 


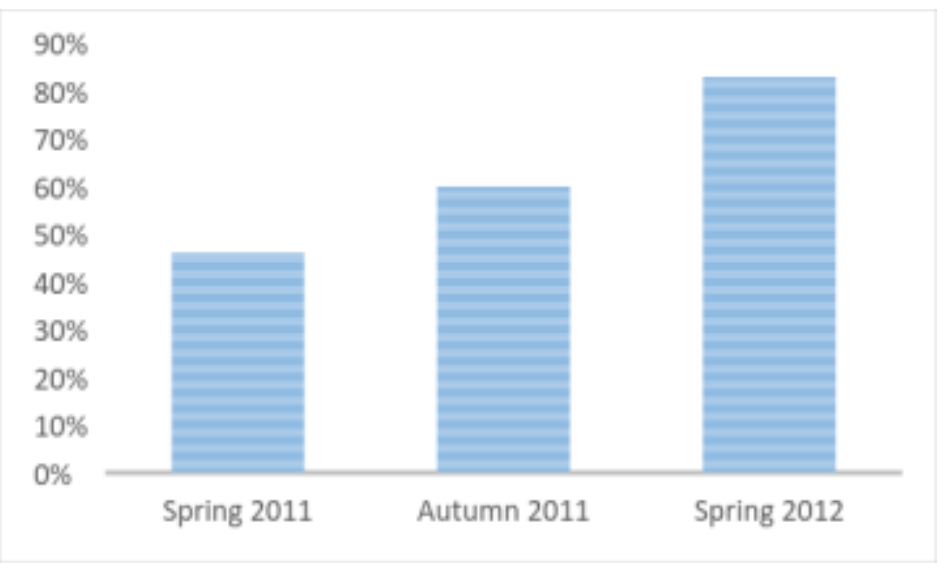

Figure 3. The number of students, who described research-based knowledge in their log entries (Excel form, June 2, 2014)

The students reflected on the importance of learning through EBP: "The importance of reading about what you see in the workplace, so that you do not just think you know what you need, but become absolutely certain" (student log, S2011), "I can clearly see the importance of having an evidence-based practice when one learns the best ways of combining new experiences with knowledge and new research. This has been an exciting task to work with, because it has given me new perspectives and experiences about how to work alone and with others to learn in the best way and as much as possible in practice" (student log, S2011), "I have become much more aware of how important it is to constantly seek new information and research in relation to being able to give the patient the best treatment. I've used textbooks, but also some online databases. From experience, I believe that one learns best through reading about what we are trying to achieve before we do it" (student log, S2011).

\section{The significance of the supervisors and LMS}

In the evaluation form, the students used a scale to evaluate the influence of supervisors ranging from 'not very satisfied' to 'excellent'.

In the spring of $2010,80 \%$ of the students answered the question relating to their level of satisfaction with the supervisors. The summarised results rated the supervisors from good to excellent in the following disaggregation (evaluation form, S2010):

- The nurse educator: $90 \%$

- The nurse supervisor: $92 \%$

- The librarian: $73 \%$.

During this work placement period (2010), the librarian was present during all the supervision meetings with two of the learning groups and visited all the groups once. The librarian also supervised the learning groups in the use of the LMS. Half of the students thought the supervision from the nurse supervisors was excellent. Supervision by the nurse educator and the librarian were rated evenly between 'good', 'very good' and 'excellent'.

The student statements may say more about how satisfied the students were with the support of the supervisors than the scaled feedback results. They expressed how they appreciated the support and help of the supervisors. To give an example "...the librarian has helped us a lot in the work with the assignment during the work placement" (student log, A2010). Another example was "Every Tuesday we have had meetings with the supervisors from the work placement, the education supervisor and the Learning Centre. We have received good support 
and learnt a lot about information seeking in Medline and Svemed+. This has been extremely informative, and we have been able to share this learning with some of the staff showing them how to conduct search techniques in databases" (student log, A2010). A third example was "When working with the assignment, we were put to the test and luckily we got great help from the Learning Centre. I will take with me all that I have learnt about information seeking in further education and the workplace" (student log, S2012). These examples of written statements show that the supervisors were accessible and significant in the students' work.

An evaluation conducted after the work placement in the autumn of 2011, indicated that most of the students rated the librarian's supervision as being of great importance, despite the fact that the librarian was only supervising in virtual format through the LMS and not at physical meetings.

The written supervision and communication through the LMS meant that every comment, downloaded document and website could be saved, shared and read over again. The students commented 59 times in the LMS, and most of them uploaded their logs and assignments.

Most of the students were positive about the supervision on the use of the LMS, essentially because it contributed to rapid help outside the physical meetings, and written guidance was clear.

In summary, the students commented frequently about the usefulness of the LMS as a mediating tool (evaluation form, S2010). They claimed they had learnt a lot of the information seeking process, because of good tips and they thought it was useful that one could see what other learning groups had done. One student even thought that it was easier to use the LMS to ask questions than to talk face-to-face to the supervisors. There was one negative comment about the LMS from a student that did not like having another system to deal with, because they already had a system on campus.

One student log commented about the LMS; "I have learnt about the importance of doing information seeking in good databases. Some databases specialise in research articles" (student $\log$, S2012).

The LMS may be of importance as a mediating factor, but the students express that they have also learnt much by exploring academic themes together, examining each other's arguments, agreeing or disagreeing and ask each other critical questions. One student described this; "Working together leads to the development of ways to think, talk, argue and interact" (student log, A2010).

The following comment says something about the importance of all three supervisors, although the librarians at the Learning Centre were only available via the LMS. "Especially in relation to the work with the assignment in the learning group, I have learnt a lot about research knowledge and how to proceed in order to seek new research. I've become better acquainted with databases such as Medline, Ovid Nursing and Svemed+; also, we have the great guidance from supervisors at work placement, the supervisor from school and from the Learning Centre. We received many great tips about how to find the right keywords and refine the search to get more specific and relevant results" (student log, S2012).

\section{Changes in preparing the students for practice periods at work placements}

As a result of the collaborative work undertaken during the clinical practice placements during the Langerud project, additional requirements in searching for research-based information were introduced as requirements for students' written assignments and were included in the new 
curriculum and student handbooks for the academic year 2012/2013 (Kolstad, 2012).

After the project ended in 2012 students' preparatory work was changed from a standalone subject course in IL and extended to a lecture about EBP. The lecture was developed in close collaboration between the nursing education department and the Learning Centre and library the EBP lecture and the course in IL were inter-supporting. Experiences from other nurse educators and from the librarian who participated in Leeway for information seeking project were used. The examples were based on real and recent experiences, which made them more relevant than those previously used. The lecture was given collaboratively by a nurse educator, a librarian and the manager of the project Leeway for information seeking.

The project initiated new ways of thinking and new interdisciplinary constellations between nurse education and the Learning Centre and library. The Learning Centre and library was invited to new meetings and projects to further develop ways in which students could be better prepared for work placements. Together, nursing educators and a librarian developed lectures that started in the autumn of 2012. These lecturers where developed for the physical classrooms. Despite the positive results of supervising through wiki and an illustrated need for this kind of tool, an LMS was not established.

\section{Discussion}

IL seems to develop through situated interaction in authentic contexts. In Kolstad (2015) IL learning is seen as taking place through communicative interaction between people and tools in the discursive practice of the work placement. General factors from a sociocultural learning perspective influenced the students' learning outcomes. These included exploring academic themes together, examining each other's arguments, agreeing or disagreeing and critical questioning. From a sociocultural perspective, IL is seen as a set of abilities to seek and use information in purposeful ways related to the task, situation and context where it happens ( $\mathrm{p}$. $58)$.

The importance of learning IL in an authentic context was most evident in the focus on work placement learning outcomes. Several results indicated that students acquired a realistic picture of the setting. Many of the students did a little research before they chose a topic for their assignment, because they wanted it to be as relevant as possible to the workplace. In some cases, the students discovered gaps themselves and started to wonder why it was different from what they had learnt on campus. They were engaged in the relevance of the information seeking process and with this their interest to find appropriate research articles increased. They were also critical of what kind of research they found. The authentic context contributed to more involvement and engagement. This was unlike working with the same assignments in the classroom. This workplace context became a 'win-win' situation for both the students and the nursing supervisors; it encouraged reflecting on why we do as we do and applying up-to-date research, according to steps three and four in evidence-based practice (Melnyk et al., 2010). The findings are supported by Coomarasamy and Khan (2004) and Sundin (2003), who found that integrated teaching produced better learning outcomes, because the students could identify real clinical problems. They also had a greater number of reflections (Cobus, 2009; Figa et al., 2009).

Kolstad (2015) found that the supervisors had different roles depending on the students' questions. The results show that the students appreciated the ability to communicate with nurses, lecturers and the librarian at the same time when they were working on their assignment. The students combined the supervisors' experiences and the knowledge they had built 
themselves, and then did the information seeking and found the most relevant research.

The results show that co-teaching in one of the learning groups in 2010 produced better IL and EBP results from the students who were participating in this department of the nursing home than later in 2011 and 2012. A nurse educator, several nurse supervisors and a librarian participated with teaching in these 2010-groups. Kolstad (2015) presents the fact hat the librarian became a 'valued member' of the supervisory team as an influencing factor. The nurse educator and librarian also agreed on the aim of the information seeking strategy to find relevant research articles and combine the information seeking competence in working in an evidencebased manner. The nurse educator was open to learn from the librarian and vice versa. Therefore, it could be that the closely shared repertoire of emphasis between the two motivated students' understanding and interest in the relevance of IL and EBP to a greater degree than the other groups (Kolstad, 2015). The characteristics of a joint supervision culture included using common terminology and concepts, required time for reflection, acknowledgement of each other's competences and support to the students.

Factors that also affected the students' results included their own initiative to share their competence in IL with the others in the learning group, and with the nurse supervisors. The IL knowledge within the group of students often varied, with some having greater competencies and experience in IL than others. The results indicated that the students shared their acquired knowledge with other members of their learning group. Generally, the fact that all the students engaged in asking questions and conducting the information seeking process several times is likely to have increased their skills. They also shared their learning process in information seeking with the nursing supervisors. Both the nurse educator and the nursing supervisors acquired increased knowledge about the information seeking process, which also benefited the students over the whole project period.

From a sociocultural perspective, EBP is seen as shaped by the demands of the practical activities in which people are regularly engaged. The EBP model was another tool used to shape workplace learning and practice. The students described the different aspects of the EBP model in a simple way, but it was a structure that students at this level could relate to (figure 1). The structure contributed to the students at this level becoming aware that this could be a way of starting to think about EBP. The EBP model became a collaborative tool that was used in all the teaching and learning settings, in meetings with the students, in the LMS and when the students worked on their assignments. The model invited and perhaps pushed the students to collaborate not just inside their learning groups, but also to learn from patients, relatives and nurses. In addition to receiving advice from the supervisory team in meetings and through the LMS, students seemed to get a more realistic view on how to work in an evidence-based manner. This was illustrated by over 60 percent of the students being able to combine aspects of the model (figure 2) and the fact that the focus on research increased significantly (figure 3 ). The positive statements from the students about EBP also confirm this awareness and interest in EBP; "I have become more aware...", "I can clearly see the importance...", "..new perspectives" (in the result section, step 5 in this article). Two studies support that both having knowledge of EBP and taking part in EBP groups are important for the development of a positive attitude to EBP (Stokke et al., 2014; Wallen et al., 2010).

An early start in thinking evidence-based should contribute to greater knowledge about IL and EBP. The students acquire positive attitudes and behaviour early on towards EBP and they can influence the integration of EBP when they finish their nursing degree programme. However, as Lloyd (2013) suggests, qualified students require some support when they start 
working, because of the rapid changes in the IL field, e. g. databases and new tools.

The findings presented in this article also confirm the importance of co-teaching on the information seeking process over a period of time; not just two hours a year on campus. In this case, during an eight week work placement period, which also provides a reality context. Over such a period, one can assume that the LMS will have an important mediating effect in how the students perform. The LMS became a virtual sociocultural learning environment. The students did have the opportunity to learn from each other by reading each other's feedback and answers to discussions on the LMS. The librarian also put written guidelines on the LMS and it became a used resource of knowledge. One can assume that the LMSs were a mediating factor in reducing inequalities between the learning groups, because all the supervisors participated in supervising the students through the LMSs, but not in the physical meetings with the students. Therefore, the differences were equalised gradually. One assumption is that the students achieved better results by having both oral and written supervision. May be the different forms of guidance brought about the positive results. The differences may be supported by varied learning styles and reinforced guidance. Over time, the students are shown to acquire a stronger focus on research (figure 3). They also expressed this through positive feedback about how they collaborated in physical meetings in the learning groups and how they appreciated the LMS and the "virtual" librarian. These findings are supported by Cobus' (2009) study that also showed a higher level of reflection and better quality assignments, because of shared pooling of experience and resources.

The LMS provided written guidance and space for exchange of experiences. It could nevertheless have been used to greater advantage if it were further developed as an established tool in guiding students in workplaces. Figa et al.'s (2009) study is one of example of research on distance students' learning outcomes when the librarian has a panel with hybrid media possibilities to do the supervision. Figas results are also supported by a study by Kraemer et al. (2007). LMSs today have hybrid facilities, because they can combine different types of supervision, assimilating face-to-face (e.g. Skype, Lync) by instructing when sharing a screen with the students, chatting, using short instruction videos and sharing documents. This will give the librarian a new chance to contribute IL competences in collaboration with both education and workplaces to multiple students, during work placements, for distance learners and for students on campus on an irregular basis.

As a result of this project, new partnerships and collaborations have been started to ensure that nurse students are better prepared for work placements. Previously preparations took place on campus, but the findings of the Leeway for information seeking project showed that the teaching and learning should rather take place in an authentic practice setting. To achieve this on an ongoing basis, it will be necessary to use co-learning on digital tools.

\section{Conclusion}

This study indicates that nurse students introduced to information seeking processes and encouraged to read research articles at an early stage in their nursing education, improved their competencies in IL and EBP significantly. This was specifically aided by learning EBP taking place and being applied in a work placement setting where they were able to describe the relevance of the research they found in real clinical situations. 
Research demonstrating positive effects of cross-collaborative or joint supervision in IL using both physical and digital channels in work placements is rare. This study provides an input, but it is an area that needs further research. One can assume that it challenges new collaborating constellations between educational programmes, academic libraries and work placement personnel, since they have to include each other in the supervision of students. This requires new organisational models that integrate all the participants in a more holistic way of thinking and acting. In Norway, the community health sector does not have specialised libraries. However, public libraries could have a role in the follow-up support of newly graduated nursing students in the community health sector.

\section{Acknowledgements}

I would especially like to thank Gerd Melsæter, the project leader for the Langerud model, who invited me to join the project and was jointly responsible for the results. In addition, I am very grateful to the participants of the Langerud project for enabling me to do this research as part of their group. I would also like to thank the Director of Learning Centre and Library, Lars Egeland, for encouraging this project to happen. This text would not have been realised without the help of my critical readers Professor Ragnar Nordlie and Senior Professor Louise Limberg. Advisor Nicola Johnston has also been indispensable who have given constructive feedback to further elaborate and improve the accessibility of this article.

\section{References}

American Psychological Association. (2010). Publication manual of the American Psychological Association (6 ${ }^{\text {th }}$ ed.). Washington, DC: Author.

Association of College \& Research Libraries. (2000). Information literacy competency standards for higher education. Retrieved February 4, 2016, from http://www.ala.org/acrl/sites/ala.org.acrl/files/content/standards/standards.pdf

Cobus, L. (2009). Using Blogs and Wikis in a graduate public health course. Medical Reference Services Quarterly, 28(1), 22-32. doi:10.1080/02763860802615922.

Coomarasamy, A., \& Khan, K. S. (2004). What is the evidence that postgraduate teaching in evidence based medicine changes anything? A systematic review. BMJ : British Medical Journal, 329(7473), 1017-1017. doi:10.1136/bmj.329.7473.1017

Dawes, M., Summerskill, W., Glasziou, P., Cartabellotta, A., Martin, J., Hopayian, K.,... Osborne, J. (2005). Sicily statement on evidence-based practice. BMC Medical Education, 5(1), 1. doi:10.1186/1472-6920-5-1

Figa, E., Bone, T., \& Macpherson, J. R. (2009). Faculty-Librarian collaboration for library services in the online classroom: Student evaluation results and recommended practices for implementation. Journal of Library \& Information Services In Distance Learning, 3(2), 67-102. doi:10.1080/15332900902979119 
Florea, M. (2008). Using WebCT, Wiki Spaces, and ePortfolios for teaching and building information literacy skills. Journal of Library Administration, 48(3/4), 411-430. doi:10.1080/01930820802289466

Kolstad, A. (2010). Evaluation form feedback from students in april-june 2010. Retrieved January 31, 2016, from https://docs.google.com/document/d/1buSmkEcKmoy7zMWj1Ik68_qQbbQWPkFLF1vZ0-LfMY/edit?usp=sharing

Kolstad, A. (2012). Bibliotekaren som samarbeidspart [The librarian as collaborative partner]. Bibliotekforum, 37(5), 30-1. Retrieved from http://norskbibliotekforening.no/wpcontent/uploads/2014/01/BF-5-2012.pdf

Kolstad, A. (2015). Co-teaching in information literacy during work placements: The libararian's role. Liber Quarterly: The Journal of European Research, 25(2), 56-86. doi:10.18352/lq.10049

Kraemer, E. W., Lombardo, S. V., \& Lepkowski, F. J. (2007). The librarian, the machine, or a little of both: A comparative study of three information literacy pedagogies at Oakland University. College \& Research Libraries, 68(4), 330-342. doi:10.5860/crl.68.4.330vv

Lloyd, A. (2013). Building Information Resilient Workers: The critical ground of workplace information literacy: what have we learnt? In S. Kurbanoğlu, E. Grassian, D. Mizrachi, R. Catts \& S. Špiranec (Eds.), Worldwide commonalities and challenges in information literacy research and practice: European Conference on Information Literacy, ECIL 2013, Istanbul, Turkey (Vol. 397, pp. 219-228). Heidelberg: Springer.

Melnyk, B. M., Fineout-Overholt, E., Stillwell, S. B., \& Williamson, K. M. (2010). EvidenceBased Practice: Step by Step: The seven steps of evidence-based practice. AJN The American Journal of Nursing, 110(1), 51-53. doi:10.1097/01.NAJ.0000366056.06605.d2

Sackett, D. L., Rosenberg, W. M. C., Gray, J. A., Haynes, R. B., \& Richardson, W. S. (1996). Evidence based medicine: what it is and what it isn't. BMJ, 312, 71-72. doi:10.1136/bmj.312.7023.71

Stokke, K., Olsen, N. R., Espehaug, B., \& Nortvedt, M. W. (2014). Evidence based practice beliefs and implementation among nurses: a cross-sectional study. BMC Nursing, 13(8). doi:10.1186/1472-6955-13-8

Sundin, O. (2003). Informationsstrategier och yrkesidentiteter: En studie av sjuksköterskors relation till fackinformation vid arbetsplatsen [Information strategies and professional identity: A study of nurses relation to trade information at the workplace] (Doctoral thesis). Gøteborgs universitet: Borås

Wallen, G. R., Mitchell, S. A., Melnyk, B., Fineout-Overholt, E., Miller-Davis, C., Yates, J., \& Hastings, C. (2010). Implementing evidence-based practice: Effectiveness of a structured multifaceted mentorship programme. Journal of Advanced Nursing, 66(12), 2761-2771. doi:10.1111/j.1365-2648.2010.05442.x 
A. Kolstad

Wenger, E. (1998). Communities of practice: Learning, meaning, and identity. Cambridge: Cambridge University Press. 